\title{
Time Precoding Enabled Non-Orthogonal Frequency Division Multiplexing
}

\author{
Waseem Ozan*, Paul Anthony Haigh ${ }^{\dagger}$, Bo Tan ${ }^{\dagger}$ and Izzat Darwazeh* \\ * Department of Electronic and Electrical Engineering, University College London, Gower Street, UK \\ $\dagger$ Intelligent Sensing and Communications Group, Newcastle University, Newcastle-upon-Tyne, UK \\ $\ddagger$ Faculty of Information Technology and Communication Sciences, Tampere University, Finland \\ i.darwazeh@ucl.ac.uk
}

\begin{abstract}
In this paper, we propose a time precoding scheme for cancelling inter-carrier interference in non-orthogonal frequency division multiplexing for the first time. Achieving high spectral efficiencies is a recurring and key challenge in wireless communications systems and researchers generally use high order and advanced modulation formats to approach this problem, in particular, non-orthogonal modulation formats are a topic of particular interest. Fast orthogonal frequency division multiplexing (F-OFDM) doubles the throughput of conventional OFDM by violating orthogonality of the quadrature carrier, causing interference in the real and imaginary domains. Here, we propose a precoding scheme that enables self-interference cancellation without the need of the interference level calculation. The proposed scheme is implemented in the context of a narrowband internet-of-things (NB-IoT) system and verified on a software define radio (SDR) testbed with realistic $A W G N$ and multiple path channels from channel emulator for concept proving. By comparing with the standard OFDM transmission, the time precoded F-OFDM outperforms around 3dB by BER with same signal-to-ratio (SNR) level.
\end{abstract}

Index Terms-Time precoding, non-orthogonal, Fast OFDM, spectrally efficient, NB-IoT waveform.

\section{INTRODUCTION}

Spectrum is one of most essential resources in any communications system, but even more so in wireless networks. Orthogonal frequency-division multiplexing (OFDM) [1] is one of the classic FDM technologies and has been widely used in $4 \mathrm{G} / 5 \mathrm{G}$ and several of $802.11 \mathrm{x}$ standards due to its ability to manage spectral nulls and maintain high throughput. Taking advantage of orthogonality between subcarriers (i.e. subcarrier separation of $1 / T$, where $T$ is the symbol period), OFDM successfully controls the inter-carrier interference (ICI). However, should this condition be violated, i.e. subcarrier separation $<1 / T$, orthogonality is violated and ICI is introduced.

Driven by increasing user density and high data rate applications, such as real time video and audio streaming, researchers have been exploring new solutions for compressing the spectrum. Fast orthogonal frequency-division multiplexing (F-OFDM) [2,3] is a subset of spectrally efficient frequency division multiplexing (SEFDM) [4,5]. F-OFDM is one important and widely discussed approach in which the spacing between subcarriers is compressed below the Nyquist limit to $1 / 2 T$.

This work was supported by the UK EPSRC grant EP/P006280/1: Multifunctional Polymer Light-Emitting Diodes with Visible Light Communications (MARVEL). WO acknowledges a UCL studentship for his PhD studies.
Unlike OFDM, F-OFDM is a non-orthogonal scheme, which packs twice as many subcarriers into the same spectrum as OFDM, thereby doubling the spectral efficiency [2]. F-OFDM's ability to save spectrum makes it a topic of increased research interest, as exemplified by the wide-ranging work that is being carried out [6-14]. However, the compression in the frequency domain causes interference exclusively in the real portion of the signal to the imaginary portion and vice versa, thus limiting the modulation to one dimension formats, such as binary phase shift keying (BPSK). Though a spectrally efficient system has been demonstrated to use a zero-forcing strategy [9] to estimate the channel, there is still strong ICI components remaining in the received signal. Thus, an effective ICI cancellation method is key to utilise fully the bandwidth saving at the same time for both the real and quadrature components of the signal. In [9], zero-forcing based waveform precoding for ICI suppression and orthogonal space precoding was used to avoid interference between antennas. Zero-forcing is a well established method to cancel out the interference, however, it results in noiseenhancement. In addition, the work in [15] implements self interference cancellation by sending a complex symbol with its precoded copy onto two neighbouring subcarriers, however, the data rate is halved.

In this paper, we propose a precoding scheme that tackles the ICI problem in the quadrature part of the signal. Inspired by Alamouti space-time block code [16] in multiple-input and multiple-output (MIMO), we leverage on the fact that F-OFDM utilises half the bandwidth of OFDM signals to counterpart the ICI in frequency domain, by generation of the image of the interference matrix. The detailed coding scheme is described in Section IV. By application of our precoding method, we demonstrate that quadrature amplitude modulation (QAM) constellations can be supported using F-OFDM whilst maintaining equivalent data rate to that of OFDM with the same order QAM. The contributions of the paper are:

- A new precoding scheme is proposed to cancel the ICI in F-OFDM without noise-enhancement.

- The ICI cancellation level is derived in analytic form.

- The time precoding non-orthogonal FDM is implemented and verified on software define radio (SDR) test-bed with realistic signal propagation environment.

- The new design demonstrates ICI cancellation in F-OFDM 
enabling QAM constellation transmission for the first time.

The rest of the paper is organised by following order: Section II introduces the signal model of traditional F-OFDM; ICI of F-OFDM is modelled in Section III; We described the time precoding in detail in Section IV; In Section V, the ICI cancelling performance of time precoding F-OFDM and final BER performance are shown with comparison with OFDM; At last the conclusion and future work are given in Section VI.

\section{FAST-OFDM SIGNAL MODEL}

In F-OFDM, thus far, symbols have been parallelised into $N$ real-valued data streams for modulation onto $N$ subcarriers spaced at $1 / 2 T$. For the same value of $N$, F-OFDM offers $50 \%$ bandwidth saving compared to OFDM at the same transmission speed and for the same number of bits/symbol. The discrete time-sampled F-OFDM symbol is, in general, expressed as [2, 3, 11]:

$$
x(k)=\frac{1}{\sqrt{Q}} \sum_{n=0}^{Q-1} S_{n} \cos \left[\frac{2 \pi n k}{2 Q}\right]
$$

where $k=\{0,1,2, \ldots, Q-1\}$ is the index of the time samples in an F-OFDM symbol, $\rho$ is the oversampling rate, $Q=\rho N$ and $S_{n}$ is the input symbol modulated on the $n^{\text {th }}$ subcarrier. Note, we include the factor of two on the numerator and denominator to highlight the factor-of-two difference with conventional OFDM [2, 3].

One of the issues with this transformation is that it neglects the quadrature portion of the Fourier transform and hence imposes the limitation for one dimensional, normally realvalued modulation formats. If one considers the quadrature part, and modulates a complex-valued signal, the transform becomes $[11,12]$ :

$$
x(k)=\frac{1}{\sqrt{Q}} \sum_{n=0}^{Q-1} S_{n} \exp \left[\frac{j 2 \pi n k}{2 Q}\right]
$$

Note, it is expected at this stage that the signal will contain significant inter-carrier interference due to the obvious loss of orthogonality.

An equivalent method for representing the discrete F-OFDM symbol is the matrix form:

$$
X=\mathbf{F} S
$$

where $X$ represents a $Q$-dimensional vector of a sampled FOFDM symbol in the time-domain, $S$ is an $Q$-dimensional vector of a complex-valued sampled input signal in the frequencydomain and $\mathbf{F}$ is a $Q \times Q$ matrix representing the sampled carrier matrix. The matrix elements of $\mathbf{F}$ are given by $\mathbf{F}_{k, n}=$ $\frac{1}{\sqrt{Q}} e^{(j \pi n k / Q)}$.

Consider that the transmitted F-OFDM symbols pass through an additive white Gaussian noise channel (AWGN), which leads to a signal contaminated by noise $Z$, and hence, the received signal is expressed as follows:

$$
\begin{aligned}
R & =\mathbf{F}^{H} X+\mathbf{F}^{H} Z=\mathbf{F}^{H} \mathbf{F} S+\mathbf{F}^{H} Z \\
& =\mathbf{C} S+Z_{F^{H}}
\end{aligned}
$$

where $R$ is the demodulated signal vector that consists of symbols and has length $Q, \mathbf{C}$ is the correlation matrix [17], $Z_{F^{H}}$ is a vector of AWGN samples correlated with the conjugate subcarriers, and $(.)^{H}$ is the transpose conjugate operation.

\section{Characterising ICI IN F-OFDM SYSTEMS}

The correlation matrix $\mathbf{C}$ quantifies the interference contribution on each subcarrier from its neighbouring subcarriers [17]. Its components can be found as:

$$
\begin{aligned}
& \mathbf{C}_{(l, n)}=\mathbf{F}_{(l, p)}^{H} \mathbf{F}_{(p, n)} \\
& =\frac{1}{Q} \sum_{p=0}^{Q-1} \exp \left[\frac{-j 2 \pi l p}{2 Q}\right] \exp \left[\frac{j 2 \pi p n}{2 Q}\right] \\
& =\frac{1}{Q} \sum_{p=0}^{Q-1} \exp \left[\frac{-j \pi(l-n) p}{Q}\right] \\
& = \begin{cases}1, & l=n \\
\frac{1}{Q} \sum_{p=0}^{Q-1} \exp \left[\frac{-j \pi(l-n) p}{Q}\right], & l \neq n\end{cases} \\
& = \begin{cases}1, & l=n \\
\frac{1}{Q}\left[\frac{1-\exp [-j \pi(l-n)]}{1-\exp \left[\frac{-j \pi(l-n)}{Q}\right]}\right], & l \neq n\end{cases}
\end{aligned}
$$

The final derivation in (5) is based on the sum of geometric series: $\sum_{k=0}^{Q-1} r^{k}=\frac{1-r}{1-r}$. From (5), we can represent the correlation matrix in summation form of two matrices as:

$$
\mathbf{C}=\mathbf{I}+\boldsymbol{\Lambda}
$$

where the matrix $\mathbf{I}$ is a $Q \times Q$ identity matrix and the matrix $\boldsymbol{\Lambda}$ carries the interference contribution on each subcarrier and is given as:

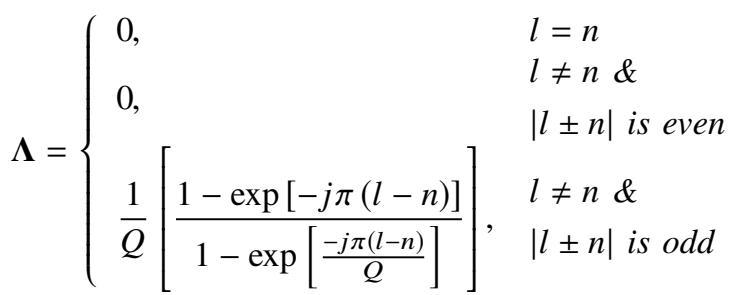

After substituting (6) in (4), the demodulated signal is represented as:

$$
\begin{aligned}
R & =\mathbf{C} S+Z_{F^{H}}=(\mathbf{I}+\boldsymbol{\Lambda}) S+Z_{F^{H}} \\
& =\mathbf{I} S+\boldsymbol{\Lambda} S+Z_{F^{H}}=S+\boldsymbol{\Lambda} S+Z_{F^{H}}
\end{aligned}
$$

where the term $\Lambda S$ indicates the additional ICI components that are added to each subcarrier.

\section{Time Precoding Self-Interference CANCEllation}

The concept of self-ICI cancellation is based on transmitting a precoded image of an original F-OFDM signal; where combining the F-OFDM signal and its image at the receiver results in self-ICI cancellation. This is the methodology that allows us to extend the modulation format to complex-valued signals for the first time. 


\section{A. Generating a precoded image}

The precoded image of the signal is generated by multiplication of the complex data symbols $(S)$ with the column vector $\Gamma$ before F-OFDM modulation using (2). At the receiver, the signal is once again multiplied with the same vector $\Gamma$, which is given as:

$$
\Gamma_{(n, 1)}= \begin{cases}1, & |n| \text { is even } \\ -1, & |n| \text { is odd }\end{cases}
$$

The received image signal of the original signal that is given in (4) is re-written and represented as:

$$
\begin{aligned}
R^{\prime} & =\Gamma \mathbf{F}^{H} X^{\prime}+\mathbf{F}^{H} Z=\Gamma \mathbf{F}^{H} \mathbf{F} \Gamma S+\mathbf{F}^{H} Z \\
& =\mathbf{C}^{\prime} S+Z_{F^{H}}
\end{aligned}
$$

\section{B. Characterizing the ICI of the precoded F-OFDM image}

In this design, we derive the F-OFDM image and its correlation matrix as follows:

$$
\begin{aligned}
& \mathbf{C}_{(l, n)}^{\prime}=\Gamma_{(l, 1)} \mathbf{F}_{(l, p)}^{H} \mathbf{F}_{(p, n)} \Gamma_{(n, 1)} \\
& =\frac{1}{Q}\left[\sum_{l=0}^{Q-1}(-1)^{l} \exp \left[\frac{-j 2 \pi l p}{2 Q}\right] \sum_{n=0}^{Q-1} \exp \left[\frac{j 2 \pi p n}{2 Q}\right](-1)^{n}\right] \\
& =\frac{1}{Q} \sum_{p=0}^{Q-1}(-1)^{l} e^{\left(\frac{-j \pi l p}{Q}\right)} e^{\left(\frac{j \pi p n}{Q}\right)}(-1)^{n} \\
& =\frac{1}{Q}(-1)^{1+n} \sum_{p=0}^{Q-1} e^{\left(\frac{-j \pi(l-n) p}{Q}\right)} \\
& = \begin{cases}1, & l=n \\
1 / Q(-1)^{1+n} \sum_{p=0}^{Q-1} e^{(-j \pi(l-n) p / Q),}, & l \neq n\end{cases} \\
& = \begin{cases}1, & l=n \\
0, & l \neq n \& \\
-1 / Q\left[\frac{1-e^{-j \pi(l-n)}}{1-e^{-j \pi(l-n)}}\right], & |l \pm n| \text { is even } \\
Q & l \neq n \& \\
& |l \pm n| \text { is odd }\end{cases} \\
& =\mathbf{I}-\boldsymbol{\Lambda}
\end{aligned}
$$

Thus, (10) is formulated as:

$$
\begin{aligned}
R^{\prime} & =\mathbf{C}^{\prime} S+Z_{F^{H}}^{\prime}=(\mathbf{I}-\mathbf{\Lambda}) S+Z_{F^{H}} \\
& =\mathbf{I} S-\boldsymbol{\Lambda} S+Z_{F H}^{\prime}=S-\boldsymbol{\Lambda} S+Z_{F^{H}}^{\prime}
\end{aligned}
$$

\section{Self-interference mechanism}

After obtaining the original F-OFDM symbol and its precoded image, we add the two symbols together. The resulting signal is therefore given as:

$$
\begin{aligned}
A & =R+R^{\prime}=S+\boldsymbol{\Lambda} S+Z_{F^{H}}+S-\boldsymbol{\Lambda} S+Z_{F^{H}}^{\prime} \\
& =2 S+Z_{F^{H}}+Z_{F^{H}}^{\prime}
\end{aligned}
$$

It should be noted that the proposed precoding ICI scheme stands based on the assumption that the channel remains invariant during one pair of original F-OFDM and its precoding image transmission period.

\section{Testbed Descriptions}

In this section we present the software and hardware designs of the real-time experiment of the proposed F-OFDM with the self-ICI cancellation mechanism. The experimental testbed contains a universal software radio peripheral (USRP) reconfigurable input-output (RIO) transceiver (National Instruments USRP-RIO 2953) programmed using LabVIEW, an Anritsu MS2830A signal analyser and a Spirent VR5 channel emulator for realistic frequency selective channels and noise generation within the analogue radio frequency (RF) domain. All of the digital signal processing blocks including the generation and detection of the signals as well as the proposed self-ISI cancellation methods are implemented in real-time. A photograph of the experimental testbed is shown in Fig. 1.

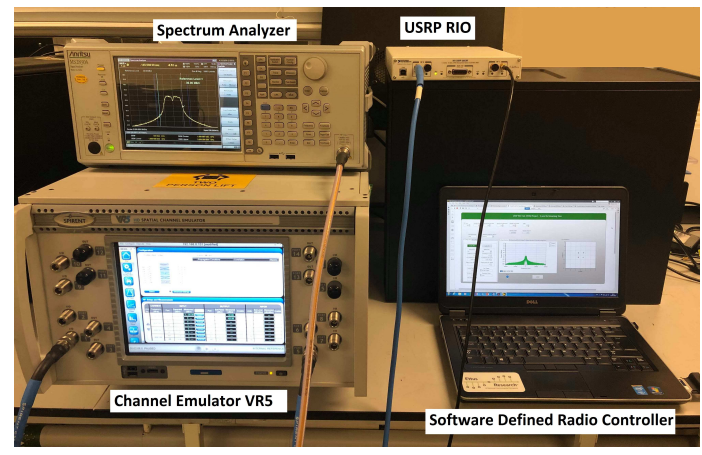

Fig. 1. Time precoding F-OFDM testbed.

\section{A. Signal generation and transmission}

At the transmitter, a pseudorandom binary sequence is generated and mapped onto the QAM constellation, with order $M$. In this experiment, we test both 4- and 16-QAM without loss of generality. Afterwards, the symbols are converted to an $\mathrm{N}$ width parallel stream via a serial-to-parallel $(S / P)$ converter which feeds an inverse fast Fourier transform (IFFT), thus generating the F-OFDM signal as depicted in Fig. 2 (the blocks at the top surrounded by the dotted green line). The frequency spacing between the subcarriers in the generated FOFDM signal is halved in comparison to that of conventional OFDM. A precoding image of the same signal is generated following the method described in section IV as shown in Fig. 2 (the blocks in the middle surrounded by dashed red line), and then the F-OFDM and its precoding image are sent using time-division multiplexing. Next, following parallel-toserial conversion $(P / S)$, and to limit the effect of inter-symbol interference (ISI) between adjacent symbols in the wireless frequency selective channel, a cyclic prefix is added at the beginning of the transmitted F-OFDM symbol. In the final transmission stage, the complex F-OFDM signal is fed into the field-programmable gate array (FPGA) that drives the USRPRIO device, to perform digital-to-analogue conversion (DAC) and up-conversion to a $2 \mathrm{GHz} \mathrm{RF}$ carrier via a local oscillator. The signal generation processes are in Fig. 2, and system parameters, which follow the narrowband internet-of-things (NB-IoT) specifications [18], are in Table I. 


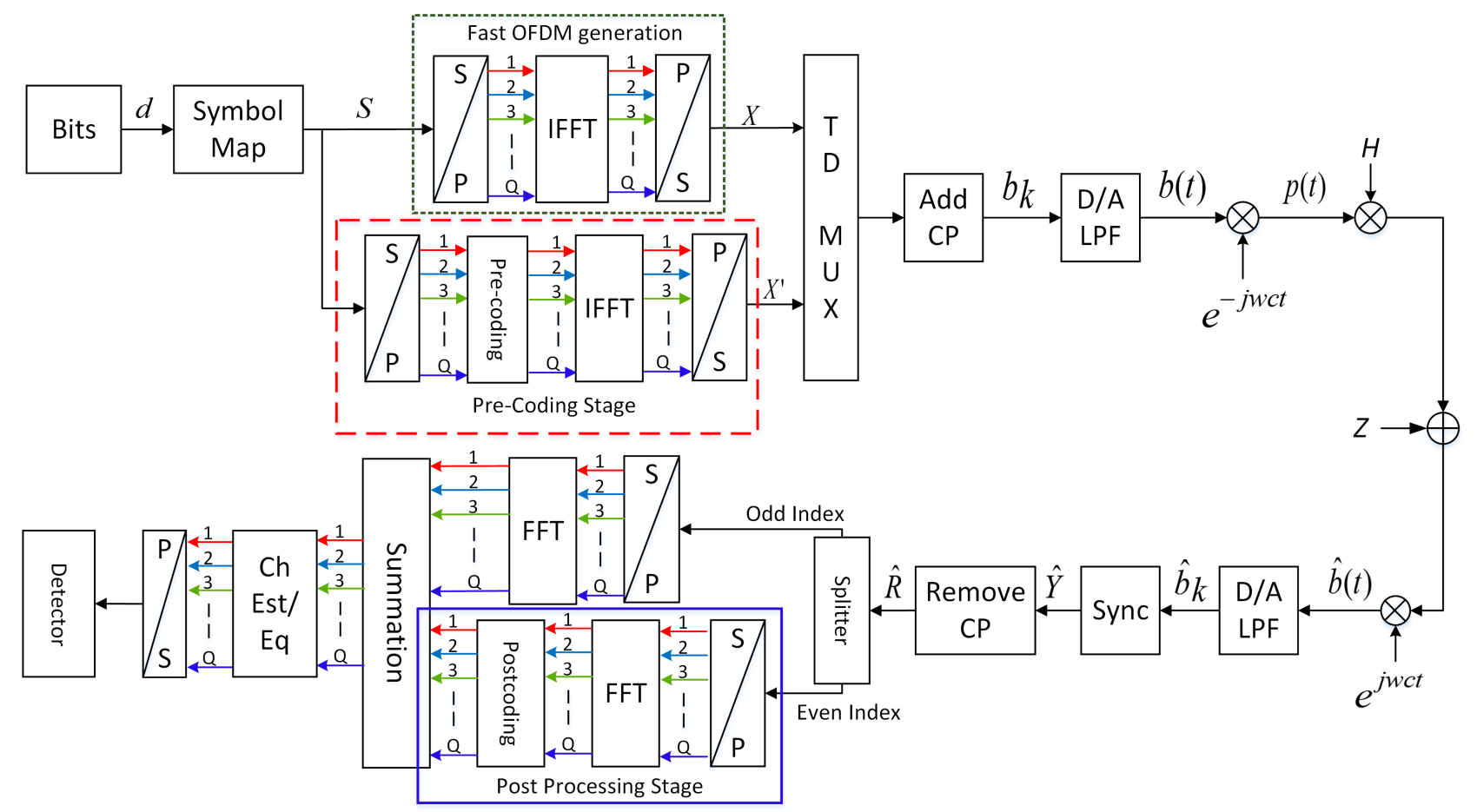

Fig. 2. Schematic block diagram of time precoding F-OFDM system.

TABLE I

EXPERIMENTAL SYSTEM SPECIFICATIONS

\begin{tabular}{lcc}
\hline Parameters & OFDM & F - OFDM \\
\hline \hline Central carrier frequency (GHz) & 2 & 2 \\
\hline Sampling frequency (MHz) & 1.92 & 1.92 \\
\hline Signal bandwidth (KHz) & 180 & 90 \\
\hline Subcarrier baseband bandwidth $(\mathrm{KHz})$ & 15 & 15 \\
\hline Subcarrier spacing $(\mathrm{KHz})$ & 15 & 7.5 \\
\hline IFFT/FFT size & 128 & 256 \\
\hline Number of cyclic prefix samples & 10 & 10 \\
\hline Number of data samples & 128 & 128 \\
\hline Number of data subcarriers & 12 & 12 \\
\hline Modulation scheme & 4-QAM & 4-QAM \\
& 16-QAM & 16-QAM \\
\hline
\end{tabular}

\section{B. Frame structure}

We use a system with frame similar to those of Long-Term Evolution (LTE) [18] where every radio frame of length $10 \mathrm{~ms}$ consists of ten equally sized subframes, each of which contains $2 \times 0.5 \mathrm{~ms}$ time slots. In every time slot, there are seven FOFDM symbols; thus, every subframe contains 14 symbols. Of these 14 symbols, two carry a pilot that is used for channel estimation and the other 12 symbols carry data signals. Fig. 3 depicts the frame structure of the transmitted signal.

\section{Frequency selective channel and signal synchronisation}

The RF frames are transmitted over a mobile environment implemented using the VR5 channel emulator. We used the LTE EPA5 wireless channel model in this work [19] (although others are applicable and have been tested but not shown here). The output of the channel emulator is fed into the USRP-RIO

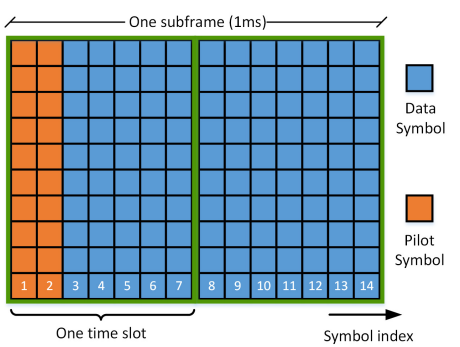

Fig. 3. Radio frame structure.

device, which down-converts the RF signal to baseband before analogue-to-digital conversion (ADC). For frame synchronisation, the popular and effective Schmidl \& Cox technique [20] is applied where two identical time sequences are attached to each radio frame.

\section{Channel estimation/equalisation and signal detection}

After CP removal, the even indexed symbols are processed as in Eq. 10 as depicted in Fig. 2 (the blocks at the bottom surrounded by the solid blue line) and then every two data symbols (i.e. the odd and the following even indexed symbol pairs) are summed to cancel the ICI between subcarriers. This summation process of the odd and even indexed symbols is given in Eq. 13. Then, channel estimation (using zero-forcing estimator in frequency domain [21]) and signal equalisation (using a one-tap equaliser in the frequency domain [21]) are applied to mitigate the channel effects. The resulting signal is free of ICI and channel effects, however, it is contaminated with 


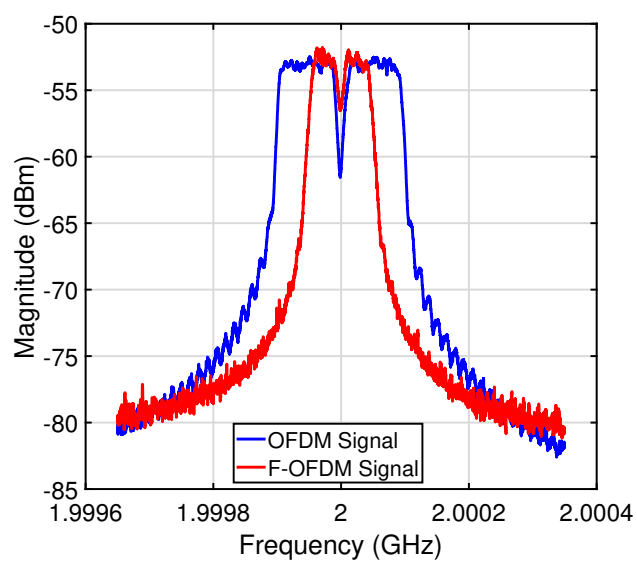

Fig. 4. Measured spectra for OFDM and F-OFDM.

the noise generated at the receiver. Therefore, a hard decision decoder is applied to recover the transmitted data.

\section{System PERFormanCE}

The frequency spectra of the two received signals, F-OFDM and OFDM, are shown in Fig. 4. In the case of using F-OFDM, only $50 \%$ of the bandwidth is required to transmit data with the same bit rate as OFDM. However, in this self-ICI cancellation F-OFDM method, we transmit the signal twice, which leads to loss of the standard F-OFDM 50\% bandwidth saving.

In Fig. 5, constellations for 4- and 16-QAM using the proposed self-ICI cancellation system are shown. From left to right, the first two constellations show the received F-OFDM signal and its precoded image, respectively. It is clear that the two signals in the constellations are highly distorted by the ICI generated between the subcarriers due to the $50 \%$ reduction in the frequency spacing between subcarriers. The third constellations indicate the results of the summation of the two constellations (i.e. the summation of F-OFDM and its precoded signal). It is evident that the ICI generated among subcarriers is cancelled in the summation process, which leads to self-ICI cancellation. Clearly, at this stage the data cannot be recovered successfully because of the channel effects and system hardware impairments, therefore, an equalisation process is required to cancel these effects. The signal after equalisation (right-most constellations) can then simply be detected using a hard decision decoder.

Finally, we measure bit error rate (BER) for a range of signal-to-noise ratios (SNRs) as a metric to evaluate the performance of the proposed self-ICI cancellation scheme and use the BER performance of conventional OFDM as a benchmark. First, we evaluate the performance in an AWGN channel. In the experimental system noise is accurately generated and added to the RF signal by the channel emulator. The 4- and 16QAM BER comparisons of OFDM and self-ICI cancellation F-OFDM are shown in Fig. 6. Second, we test the system performance across the (frequency selective, multi-path) LTE EPA5 channel contaminated with AWGN noise. The 4- and 16-QAM BER performances of both OFDM and F-OFDM

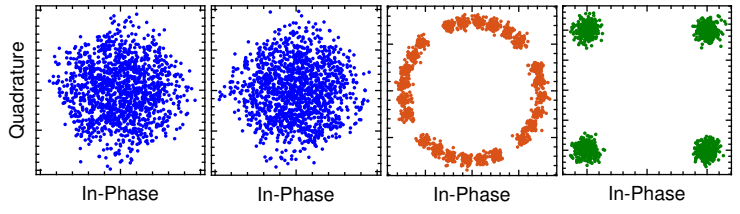

(a) 4-QAM modulation

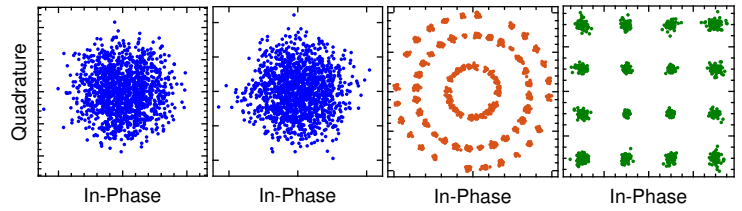

(b) 16-QAM modulation

Fig. 5. Received constellations for 4-QAM and 16-QAM signals. For both row (a) and (b), from left to right, the first two constellations show the received F-OFDM and precoded signals; the third shows the summation of the two signals; and the most right constellation shows the signal after equalisation process.

with self-ICI cancellation are shown in Fig. 7. The results in Fig. 6, prove that the simple addition process of the original F-OFDM signal and its precoding image at the receiver is sufficient to cancel the ICI between the subcarriers caused by the compression in signal bandwidth, and hence enable data recovery when using complex modulation formats for the first time. The BER performance shown in Fig. 7 indicates that the multi-path effects degrade the performance of both systems under test, where the $\sim 3 \mathrm{~dB}$ performance gap (i.e. in SNR) is maintained for either the AWGN or noisy multi-path channel. The reason for this SNR gap is simply that the time precoding F-OFDM transmits the signal twice. The first transmission is the original F-OFDM signal and the second is the precoding copy of the same signal. Therefore, the signal power at the receiver adds up, while the ICI of the two signals is mutually cancelled. Hence, the proposed F-OFDM method demonstrates a $3 \mathrm{~dB}$ SNR advantage over OFDM.

\section{CONCLUSION}

In this work, we proposed a time precoding method for cancelling out the ICI in F-OFDM transmission. The analytic ICI cancelling process of the proposed method is provided and the performance is assessed with experimental SDR system and BER measurements. The proposed time precoding and self ICI cancelling method is experimentally shown to be effective in both AWGN and frequency selective multi-path channels, thus paving the way for the practical use of F-OFDM in wireless data transmission. Furthermore, this work will hopefully inspire future exploration of spatial and joint time-space precoding for non-orthogonal multicarrier system, which may improve the spectral and energy efficiency of wireless communication standards.

\section{REFERENCES}

[1] Y. S. Cho; J. Kim; W. Y. Yang; C. G. Kang, "Introduction to OFDM," in MIMO-OFDM Wireless Communications with MATLAB, , IEEE, 2010. 


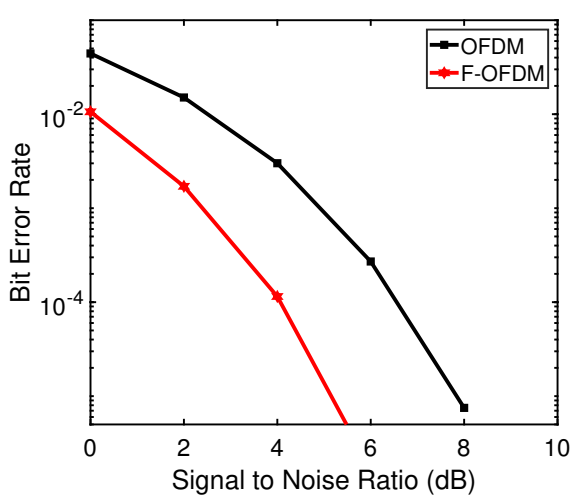

(a) 4-QAM modulation

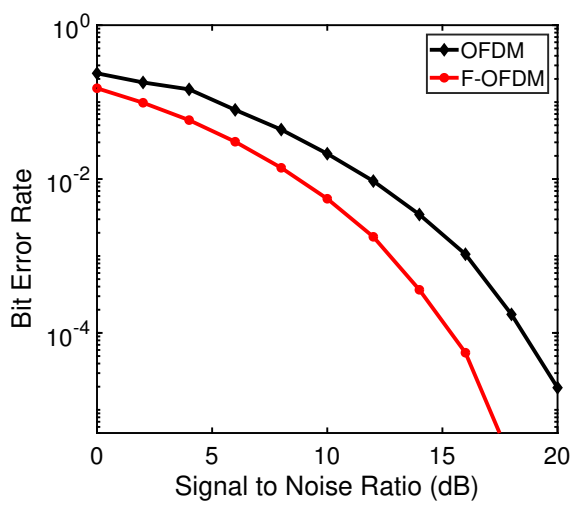

(b) 16-QAM modulation

Fig. 6. Experimental BER of 4-QAM and 16-QAM for OFDM and F-OFDM signals in AWGN channel.

[2] M. Rodrigues and I. Darwazeh, "Fast OFDM: a proposal for doubling the data rate of OFDM schemes," in International conference on Telecommunications, vol. 3, 2002.

[3] K. Li "Fast orthogonal frequency division multiplexing (Fast-OFDM) for wireless communications", PhD Thesis, UCL, 2008.

[4] I. Kanaras, A. Chorti, M. R. D. Rodrigues and I. Darwazeh, "Spectrally Efficient FDM Signals: Bandwidth Gain at the Expense of Receiver Complexity,“. Communications, 2009. ICC '09. IEEE International Conference on, Dresden, 2009, pp. 1-6.

[5] I. Darwazeh, H. Ghannam and T. Xu, "The First 15 Years of SEFDM: A Brief Survey," 2018 11th International Symposium on Communication Systems, Networks \& Digital Signal Processing (CSNDSP), Budapest.

[6] T. Xu and I. Darwazeh, "Non-Orthogonal Narrowband Internet of Things: A Design for Saving Bandwidth and Doubling the Number of Connected Devices," in IEEE Internet of Things Journal, vol. 5, no. 3, pp. 21202129, June 2018.

[7] T. Xu and I. Darwazeh, "Non-Orthogonal Waveform Scheduling for Next Generation Narrowband IoT," 2018 IEEE Globecom Workshops (GC Wkshps), Abu Dhabi, United Arab Emirates, 2018, pp. 1-6.

[8] S. K. Ibrahim, J. Zhao, D. Rafique, J. A. O'Dowd and A. D. Ellis, "Demonstration of world-first experimental optical Fast OFDM system at 7.174Gbit/s and 14.348Gbit/s," 36th European Conference and Exhibition on Optical Communication, Torino, 2010, pp. 1-3.

[9] T. Xu, C. Masouros and I. Darwazeh, "Waveform and Space Precoding for Next Generation Downlink Narrowband IoT," in IEEE Internet of Things Journal.

[10] Z. Hu and C. Chan, "A 7-D Hyperchaotic System-Based Encryption Scheme for Secure Fast-OFDM-PON," in Journal of Lightwave Technology, vol. 36, no. 16, pp. 3373-3381, 15 Aug.15, 2018.

[11] P. Anthony Haigh and I. Darwazeh, "Visible light communications: Fastorthogonal frequency division multiplexing in highly band limited con-

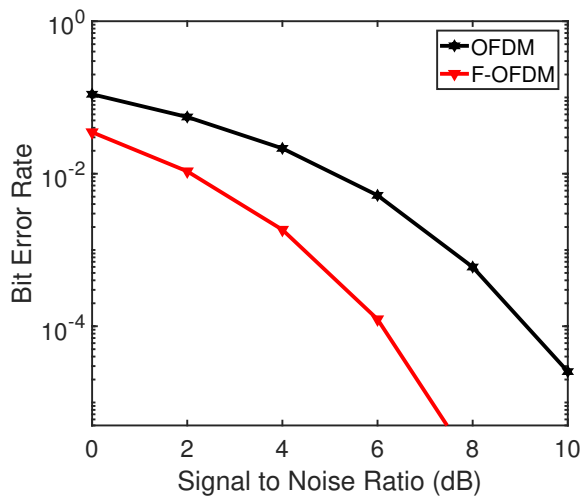

(a) 4-QAM modulation

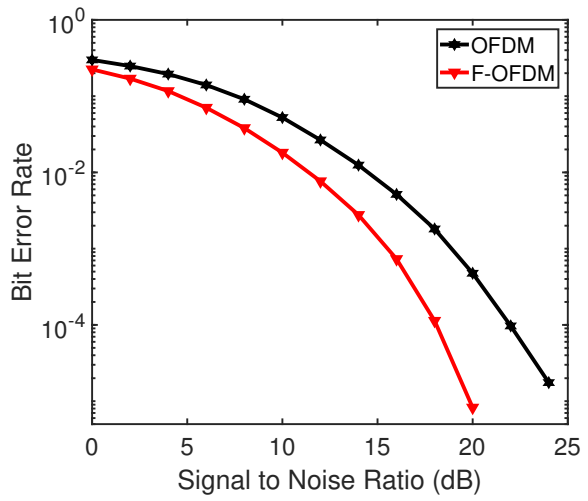

(b) 16-QAM modulation

Fig. 7. Experimental BER of 4-QAM and 16-QAM for OFDM and F-OFDM signals in LTE EPA5 channel.

ditions," 2017 IEEE/CIC International Conference on Communications in China (ICCC Workshops), Qingdao, 2017, pp. 1-8.

[12] Z. Hu, J. Zhao, Y. Hong, S. Gao, C. Chan and L. Chen, "SetPartitioned QAM Fast-OFDM with Real-Valued Orthogonal Circulant Matrix Transform Pre-Coding," 2018 Conference on Lasers and ElectroOptics (CLEO), San Jose, CA, 2018, pp. 1-2.

[13] W. Ozan, P. A. Haigh, B. Tan and I. Darwazeh, "Experimental SEFDM Pipelined Iterative Detection Architecture with Improved Throughput," IEEE 87th Vehicular Technology Conference (VTC Spring), Porto, 2018.

[14] F.-L. Luo and C. Zhang, Signal Processing for 5G: Algorithms and Implementations. Wiley, 2016.

[15] T. Xu and I. Darwazeh, "Experimental Validations on Self Interference Cancelled Non-Orthogonal SEFDM Signals," 2018 IEEE 87th Vehicular Technology Conference (VTC Spring), Porto, 2018, pp. 1-5.

[16] S. Alamouti, "A simple transmit diversity technique for wireless communications", IEEE J. Sel. Areas Commun., vol. 16, no. 8, Oct. 1998.

[17] S. Isam and I. Darwazeh, "Characterizing the intercarrier interference of non-orthogonal Spectrally Efficient FDM system," 2012 8th International Symposium on Communication Systems, Networks \& Digital Signal Processing (CSNDSP), Poznan, 2012, pp. 1-5.

[18] LTE; Evolved Universal Terrestrial Radio Access (E-UTRA); Physical Layer Procedures, Rel. 14, 3GPP Standard v.14.2.0, Apr. 2017.

[19] Evolved Universal Terrestrial Radio Access (E-UTRA); Base station radio transmission and reception, 3GPP TS 36.104 V10.2.0, May 2011.

[20] T. M. Schmidl and D. C. Cox, "Robust frequency and timing synchronization for OFDM," in IEEE Transactions on Communications, vol. 45, no. 12, pp. 1613-1621, Dec 1997.

[21] S. Boumard and A. Mammela, "Channel estimation versus equalization in an OFDM WLAN system," IEEE Vehicular Technology Conference, Spring 2001. Proceedings (Cat. No.01CH37202), Rhodes, Greece, 2001. 